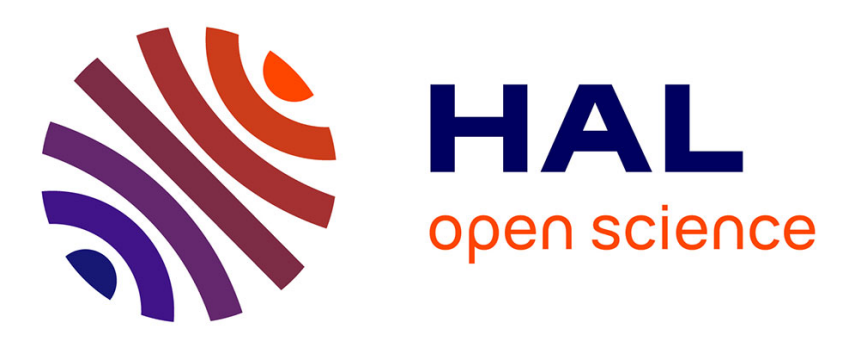

\title{
Distributed energy-efficient power optimization for relay-aided heterogeneous networks
}

Ivan Stupia, Luc Vandendorpe, Luca Sanguinetti, Giacomo Bacci

\section{To cite this version:}

Ivan Stupia, Luc Vandendorpe, Luca Sanguinetti, Giacomo Bacci. Distributed energy-efficient power optimization for relay-aided heterogeneous networks. International Workshop on Wireless Networks (WiOpt - WCN), May 2014, Hammamet, Tunisia. pp.563 - 568, 10.1109/WIOPT.2014.6850347. hal-01098833

\section{HAL Id: hal-01098833 https://hal.science/hal-01098833}

Submitted on 29 Dec 2014

HAL is a multi-disciplinary open access archive for the deposit and dissemination of scientific research documents, whether they are published or not. The documents may come from teaching and research institutions in France or abroad, or from public or private research centers.
L'archive ouverte pluridisciplinaire HAL, est destinée au dépôt et à la diffusion de documents scientifiques de niveau recherche, publiés ou non, émanant des établissements d'enseignement et de recherche français ou étrangers, des laboratoires publics ou privés. 


\section{Distributed Energy-Efficient Power Optimization for Relay-Aided Heterogeneous Networks}

\author{
Ivan Stupia and Luc Vandendorpe \\ ICTEAM Institute \\ Université Catholique de Louvain \\ B-1348 Louvain-la-Neuve, Belgium
}

\author{
(Invited Paper)
}

\author{
Luca Sanguinetti and Giacomo Bacci \\ Dip. Ingegneria dell'Informazione, University of Pisa, Italy \\ and Consorzio Nazionale Interuniversitario per \\ le Telecomunicazioni (CNIT), Parma, Italy
}

\begin{abstract}
This paper presents an energy-efficient power allocation for relay-aided heterogeneous networks subject to coupling convex constraints, that make the problem at hand a generalized Nash equilibrium problem. The solution to the resource allocation problem is derived using a sequential penalty approach based on the advanced theory of quasi variational inequality, which allows the network to converge to its generalized Nash equilibrium in a distributed manner. The main feature of the proposed approach is its decomposability, which leads to a two-layer distributed algorithm with provable convergence.
\end{abstract}

\section{INTRODUCTION}

The wireless research community has deployed a massive effort to study and design transmission techniques taking into account the cost of energy in wireless communications [1]. Towards this end, the concept of link capacity per unit cost originally proposed in [2] has been widely adopted in many different contexts. Just to name few examples, the problem of energy efficient precoding for wireless terminals equipped with multiple antenna capability is studied in [3], whereas [4] and [5] investigate energy efficient link adaptation strategies for wideband systems.

From an architectural point of view, the hypothesis of improving the energy efficiency (EE) performance through a very dense deployment of self-organizing, low-cost, and low-power base stations has been investigated in [6]. This perspective has stimulated an intense research activity on heterogeneous networks, which are founded on the idea of multiple radio access technologies and transmission techniques coexisting in the same area to ensure the most efficient usage of the spectrum resource with the minimum waste of energy [7].

A common way of designing such heterogeneous multiuser systems is by optimizing the (weighted) sum of the users objective functions. Despite its promise, solution methods based on "socially-efficient" optimization are often too demanding, the main difficulty lying in adopting distributed techniques

The research leading to these results has received funding from the European Commission in the framework of the FP7 Network of Excellence in Wireless COMmunications NEWCOM\# (Grant agreement no. 318306), from the People Programme (Marie Curie Actions) of the European Union's FP7 under REA Grant agreements no. PIOF-GA-2011-302520 GRAND-CRU and PIEF-GA-2012-330731 Dense4Green, from the Belgian Science Policy Office under the IAP 7/23 BESTCOM project, and from the Belgian Fonds de la Recherche Scientifique (FNRS). with limited signaling among the users. On the contrary, gametheoretical approaches based on competition among users lead to alternative distributed algorithms that present advantages in term of robustness of convergence, scalability, and required quantity of message passing [8]. In this perspective, the design of wireless devices capable of self-enforcing the negotiated agreements on the resource usage has been investigated in [9]-[11]. In [9], the authors proposed an accurate analysis of the equilibrium point for a group of competitive users aiming at maximizing their information rate in parallel Gaussian multi access channels. However, this capacity-based approach is not energy-efficient since the selfish behavior of players tends to increase transmit power beyond what is reasonable. To overcome this obstacle, in [10] a power allocation game was proposed to maximize the EE of the users' links. Although interesting, the framework developed in [10] does not provide a theoretically convergent algorithm. This gap has been recently fulfilled in [11] by making use of the quasi variational inequality (QVI) framework that was originally introduced in [12] and successfully used in different fields such as economics and biology (see [13] and references therein). The main objective of this work is to apply the approach proposed in [11] to relay-aided heterogenous networks in which different types of users may pursue different objectives. Moreover, minimum performance requirements will be guaranteed by imposing feasible strategy sets including also coupling convex constraints.

Notation: Matrices and vectors are denoted by bold letters: $\mathbf{I}_{L}, \mathbf{0}_{L}$, and $\mathbf{1}_{L}$ are the $L \times L$ identity matrix, the $L \times 1$ all-zero column vector, and the $L \times 1$ all-one column vector, respectively. The set of positive real numbers (including 0 ) is denoted by $\mathbb{R}_{+}$, and $(\cdot)^{T}$ denotes transposition. The notation $[x]^{+}$stands for $\max (0, x), W(\cdot)$ denotes the Lambert $W$ function [14], defined to be the multivalued inverse of the function $z=W(z) e^{W(z)}$ for any $z \in \mathbb{C}$, with $\mathbb{C}$ being the set of complex numbers, and $\mathbb{E}\{\cdot\}$ denotes statistical expectation.

\section{SySTEM SCENARIO}

Two-tier networks are a promising solution to reduce the energy consumption in cellular networks. Operationally, in a two-tier network small cells are integrated into existing macro-cellular networks, so that the latter can ensure wide-area 
coverage, whereas the small cells can carry most of the data traffic for low-mobility users within their short radio coverage.

More specifically, we consider the uplink of the two-tier network depicted in Fig. 1 composed by one macro base station (MBS) serving $M$ macro users (MUs), by a number of small cells serving a total of $S$ small-cell users (SCUs), and $R$ relay stations (RSs), each serving at most one MU. The RSs are assumed to be fixed and used to forward the received signal to the MBS by means of a regenerative decode-and-forward (DF) strategy. The total number of users in the network is thus given by $K=M+S+R$. In addition to this, we call:

1) $\mathcal{M}_{1}$ the set of MUs whose communication with the MBS takes place in a single hop;

2) $\mathcal{M}_{2}$ the set of MUs whose communication with the MBS takes place in two hops (with the aid of relays);

3) $\mathcal{S}$ the set of SCUs that communicate with their associated small-cell base station;

4) $\mathcal{R}$ the set of RSs that communicate with the MBS.

We consider a two-time-slot transmission pattern, which is half-duplex in the sense that transmission and reception at the RSs do not occur simultaneously in the same frequency band. During the first time slot, the nodes belonging to the set $\mathcal{I}_{1} \triangleq\left\{\mathcal{M}_{1} \cup \mathcal{M}_{2} \cup \mathcal{S}\right\}$ are active, while the RSs are silent. During the second time slot, the relays decode the messages of the two-hop MUs and forward them to the MBS while the two-hop MUs stay in the idle mode. This means that the set of active users during the second time-slot is $\mathcal{I}_{2} \triangleq\left\{\mathcal{M}_{1} \cup \mathcal{R} \cup \mathcal{S}\right\}$. We assume that the direct link between two-hop MUs and MBS is negligible due to large path-loss attenuation.

The performance achieved by a generic node $k$ is measured by a two-dimensional payoff (utility) vector $\mathbf{u}_{k}=$ $\left[u_{k, 1}, u_{k, 2}\right]^{T}$, in which $u_{k, 1}$ and $u_{k, 2}$ denote the value of the $k$ th objective function during the first and second time slots, respectively. Given the different classes of users populating the two-tier network described above, the utility $u_{k, i}$ achieved by the $k$ th transmitter at the $i$ th time slot depends on user $k$ 's type (as better detailed in the next section).

More in details, assuming to have a multicarrier system with $N$ subchannels, each user can maximize its own payoff by regulating its own transmit power vector $\mathbf{p}_{k, i}=$ $\left[p_{k, i}(1), p_{k, i}(2), \ldots, p_{k, i}(N)\right]^{T}$, where $p_{k, i}(n) \geq 0$ denotes node $k$ transmit power over subchannel $n \in\{1, \ldots, N\}$ and time slot $i \in\{1,2\} .{ }^{1}$ In doing so, only local channel state information (CSI) is assumed to be available at node $k$. This amounts to saying that the $k$ th node has only knowledge of $H_{k, k}(n)$ for $n=1,2, \ldots, N$, where $H_{j, k}(n)$ is the channel transfer function over the $n$th subchannel between transmitter $j$ and transmitter $k$ 's receiver.

As is known, the optimization of the network through centralized power control strategies is extremely challenging, as it requires the coordination among heterogeneous entities, thus making its solution weakly scalable and adaptive [8]. A possible way to overcome this obstacle is to make use of

\footnotetext{
${ }^{1}$ Using the notation introduced above, it follows that $\mathbf{p}_{k, 1}=\mathbf{0}_{N}$ if $k \in \mathcal{R}$, and, analogously, $\mathbf{p}_{k, 2}=\mathbf{0}_{N}$ if $k \in \mathcal{M}_{2}$.
}

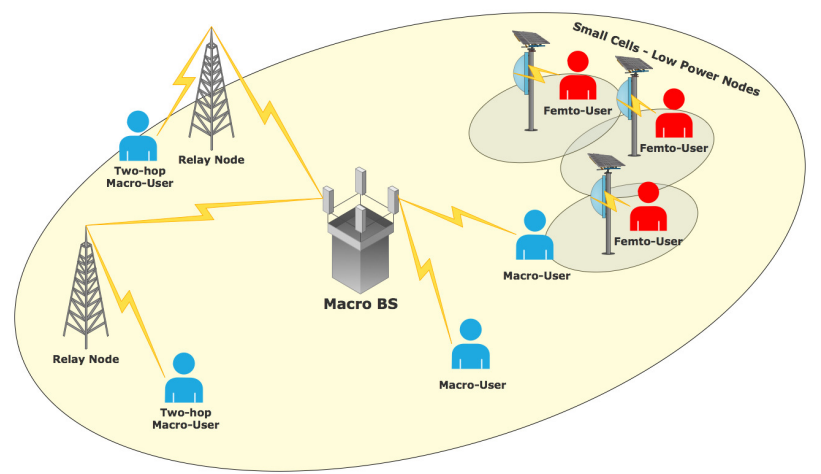

Fig. 1: Two-tier relay-assisted wireless network.

distributed (albeit suboptimal) algorithms based on a noncooperative game theoretical approach (e.g., [9], [15]).

\section{ANALYSIS OF THE GAME}

The aim of this section is to study the power control problem as the solution of a non-cooperative game $\mathcal{G}=$ $\left(\mathcal{K},\left\{\mathcal{A}_{k}\right\},\left\{\mathbf{u}_{k}\right\}\right)$, where the set of users $\mathcal{K}$ and user $k$ 's utility $\mathbf{u}_{k}$ have been introduced in Sect. II, and $\mathcal{A}_{k} \subseteq \mathbb{R}_{+}^{2 N}$ is the power strategy set available to each user $k$. Aiming at studying the solution of $\mathcal{G}$, the best response for each type of players is first derived and then used to define a QVI problem whose solution will coincide with the equilibrium of the game [11].

\section{A. Macro-cell users}

Following the aforementioned non-cooperative approach, we assume that MUs (at each time step $i$ ) target the maximization of their own information rates under a constraint on the maximum total power consumption. Otherwise stated, the best response $\mathcal{B}_{k, i}(\cdot)$ taken by a MU user $k \in\left\{\mathcal{M}_{1} \cup \mathcal{M}_{2}\right\}$ to a specific power allocation profile $\mathbf{p}_{\backslash k, i}=\mathbf{p}_{i} \backslash \mathbf{p}_{k, i}=\left[\mathbf{p}_{1, i}^{T}, \ldots, \mathbf{p}_{k-1, i}^{T}, \mathbf{p}_{k+1, i}^{T}, \ldots, \mathbf{p}_{K, i}^{T}\right]^{T}$, with $\mathbf{p}_{i}=\left[\mathbf{p}_{1, i}^{T}, \ldots, \mathbf{p}_{K, i}^{T}\right]^{T}$, can be expressed as a function $\mathcal{B}_{k, i}: \mathbb{R}_{+}^{N(K-1)} \rightarrow \mathbb{R}_{+}^{N}$, such that [11]

$$
\mathcal{B}_{k, i}\left(\mathbf{p}_{\backslash k, i}\right)=\underset{\mathbf{p}_{k, i} \in \mathbb{R}_{+}^{N}}{\arg \max } u_{k, i}\left(\mathbf{p}_{i}\right) \quad \text { s.t. } \mathbf{1}_{N}^{T} \mathbf{p}_{k, i} \leq P_{k, i}
$$

with $P_{k, i}$ being user $k$ 's maximum available total transmit power during time slot $i$ and such that $P_{k, i}>0 \forall k \in \mathcal{M}_{1}$ and $\forall i \in\{1,2\}$, and $P_{k, 1}>0$ and $P_{k, 2}=0 \forall k \in \mathcal{M}_{2}$, respectively. In addition, $u_{k, i}\left(\mathbf{p}_{i}\right)$ denotes the user $k$ 's utility function, which is given by

$$
u_{k, i}\left(\mathbf{p}_{i}\right)=R_{k}\left(\mathbf{p}_{i}\right)=\sum_{n=1}^{N} \log _{2}\left(1+\varphi_{k, i}(n) p_{k, i}(n)\right)
$$

where $R_{k}\left(\mathbf{p}_{i}\right)$ is the achievable information rate on the link between the $k$ th MU and the MBS, and

$$
\varphi_{k, i}(n)=\frac{\left|H_{k, k}(n)\right|^{2}}{\sigma_{k}^{2}(n)+\sum_{j \neq k}\left|H_{j, k}(n)\right|^{2} p_{j, i}(n)}
$$


denotes user $k$ 's equivalent channel gain over subchannel $n$, which accounts for the AWGN power $\sigma_{k}^{2}(n)$ and the multiaccess interference. The set of feasible strategies for the MUs is then given by $\mathcal{A}_{k}=\mathcal{A}_{k, 1} \times \mathcal{A}_{k, 2}$ with

$$
\mathcal{A}_{k, i}=\left\{\mathbf{p}_{k, i} \in \mathbb{R}_{+}^{N}: \mathbf{1}_{N}^{T} \mathbf{p}_{k, i} \leq P_{k, i}\right\} .
$$

Using [9], the best response (1) is given by the waterfilling mapping

$$
\mathcal{B}_{k, i}\left(\mathbf{p}_{\backslash k, i}\right)=\mathrm{wf}_{k, i}\left(\mathbf{p}_{\backslash k, i}, \mu_{k, i}\right)
$$

where the $n$th component of the waterfilling operator is

$$
\left[\mathrm{wf}_{k, i}\left(\mathbf{p}_{\backslash k, i}, \mu_{k, i}\right)\right]_{n}=\left[\frac{1}{\mu_{k, i}}-\frac{1}{\varphi_{k, i}(n)}\right]^{+}
$$

and $\mu_{k, i}$ is such that $\mathbf{1}_{N}^{T} \mathbf{p}_{k, i}=P_{k, i}$. Note that if $P_{k, 2}=0$ then $\left[\mathcal{B}_{k, 2}\left(\mathbf{p}_{\backslash k, 2}\right)\right]_{n}=0$.

\section{B. Small-cell users}

When dealing with SCUs, not only the rate maximization, but also the interference reduction between macro-cell and small-cell networks comes into play. Consequently, the best response taken by each user $k \in \mathcal{S}$ can still be expressed by (1), but with a different utility function, defined as

$$
u_{k, i}\left(\mathbf{p}_{i}\right)=E_{k}\left(\mathbf{p}_{i}\right)=\frac{R_{k}\left(\mathbf{p}_{i}\right)}{\Psi_{k}+\mathbf{1}_{N}^{T} \mathbf{p}_{k, i}}
$$

where $R_{k}\left(\mathbf{p}_{i}\right)$ is given in (2), $\Psi_{k}$ is the (non-radiative) circuit power consumed at transmitter $k$ [5], [10], and the set of feasible powers is defined as in (4).

Adapting the results derived in [16] for the problem at hand, user $k \in \mathcal{S}$ 's best response $\mathcal{B}_{k, i}\left(\mathbf{p}_{\backslash k, i}\right)$ can be still expressed by the waterfilling operator (5), provided that the water level $\mu_{k, i}$ is replaced by

$$
\lambda_{k, i}=\max \left\{\mu_{k, i}, \nu_{k, i}\right\}
$$

where $\mu_{k, i}$ is such that $\mathbf{1}_{N}^{T} \mathbf{p}_{k, i}=P_{k, i}$ whereas

$$
\nu_{k, i}=e^{\left(\beta_{k, i}-1\right)-W\left(\alpha_{k, i} \cdot e^{\beta_{k, i}-1}\right)}
$$

represents the water level that provides the maximum achievable EE, measured as in (7), for a given interference allocation $\mathbf{p}_{\backslash k, i}$ without any constraint. In addition,

$$
\begin{aligned}
\alpha_{k, i} & =\frac{1}{N}\left(\Psi_{k}-\sum_{n=1}^{N} \frac{1}{\varphi_{k, i}(n)}\right) \\
\beta_{k, i} & =\frac{1}{N} \sum_{n=1}^{N} \ln \left(\varphi_{k, i}(n)\right) .
\end{aligned}
$$

Following the same line of reasoning proposed in [11], a key ingredient to reformulate the power control problem as a solvable QVI is the mapping $t_{k, i}: \mathbb{R}_{+}^{N(K-1)} \rightarrow \mathbb{R}_{+}$, providing the inverse of the total power dissipation when $P_{k, i}$ tends to infinity, and defined as

$$
t_{k, i}\left(\mathbf{p}_{\backslash k, i}\right)=\frac{1}{\Psi_{k}+\mathbf{1}_{N}^{T} \mathbf{z}_{k, i}\left(\nu_{k, i}\right)}
$$

where

$$
\mathbf{z}_{k}\left(\nu_{k, i}\right)=\mathrm{wf}_{k, i}\left(\mathbf{p}_{\backslash k, i}, \nu_{k, i}\right)
$$

with $\nu_{k, i}$ defined as in (9) and such that the condition

$$
R_{k}\left(\mathbf{z}_{k, i}\left(\nu_{k, i}\right), \mathbf{p}_{\backslash k, i}\right)-\nu_{k, i}\left(\Psi_{k}+\mathbf{1}_{N}^{T} \mathbf{z}_{k, i}\left(\nu_{k, i}\right)\right)=0 .
$$

is satisfied [5]. Hence, combining (7) with (14), $\nu_{k, i}$ is found to explicitly represent the unconstrained $\mathrm{EE}$ achievable by a user $k \in \mathcal{S}$ for a given $\mathbf{p}_{\backslash k, i}$.

\section{Relay nodes}

In the spirit of greening the network infrastructures, the powers selected by the RSs are also dynamically adapted to maximize the EE of the relay-destination transmission, similarly to what considered by SCUs. RSs might also guarantee some minimum performance in terms of rates to the two-hop MUs, by keeping their information rate above a threshold. Hence, the best response taken by a $\mathrm{RS} k \in \mathcal{R}$ is in the form ${ }^{2}$

$$
\begin{aligned}
\mathcal{B}_{k, 2}\left(\mathbf{p}_{1} ; \mathbf{p}_{\backslash k, 2}\right)=\underset{\mathbf{p}_{k, 2} \in \mathbb{R}_{+}^{N}}{\arg \max } & u_{k, 2}\left(\mathbf{p}_{2}\right) \\
\text { s.t. } \quad & \mathbf{1}_{N}^{T} \mathbf{p}_{k, 2} \leq P_{k, 2} \\
& R_{k}\left(\mathbf{p}_{2}\right) \geq R_{\pi(k)}\left(\mathbf{p}_{1}\right)
\end{aligned}
$$

where the utility $u_{k, 2}\left(\mathbf{p}_{2}\right)$ is defined as in (7), the constraint (16) accounts for the maximum total power $P_{k, 2}$, whereas the constraint (17) is introduced to guarantee a minimum rate to the MU $\pi(k)$ served by the RS $k$, with $\pi: \mathcal{R} \rightarrow \mathcal{M}_{2}$ being the function that associates the relay $k$ with its served MU $\pi(k) \in \mathcal{M}_{2}$. The set of feasible strategies for the RSs is then given by

$\mathcal{A}_{k, 2}=\left\{\mathbf{p}_{k, 2} \in \mathbb{R}_{+}^{N}: \mathbf{1}_{N}^{T} \mathbf{p}_{k, 2} \leq P_{k, 2}, R_{k}\left(\mathbf{p}_{2}\right) \geq R_{\pi(k)}\left(\mathbf{p}_{1}\right)\right\}$.

Note that, since (17) depends on the power allocation $\mathbf{p}_{1}$ taken in the first time slot, RS $k$ 's best response is a function $\mathcal{B}_{k, 2}$ : $\mathbb{R}_{+}^{N(2 K-1)} \rightarrow \mathbb{R}_{+}^{N}$, which depends on both $\mathbf{p}_{1}$ and $\mathbf{p}_{\backslash k, 2}{ }^{3}$

Using the results in [5] and [16], similarly to the case reported in Sect. III-B, (15) can be computed in a closed form using (5), with a water level

$$
\lambda_{k, 2}=\min \left\{\nu_{k, 2}, \xi_{k, 2}\right\}
$$

where $\nu_{k, 2}$ is defined as in (9), and

$$
\xi_{k, 2}=\sqrt[N]{\frac{1}{2^{R_{\pi(k)}\left(\mathbf{p}_{1}\right)}} \prod_{n=1}^{N} \varphi_{k, 2}(n)}
$$

\footnotetext{
${ }^{2}$ Since RSs are not active in the first time slot, we report all quantities for the second time slot $i=2$ only.

${ }^{3}$ For the sake of notation, from now on we will harmonize the best-response functions for all users in the network, such that $\mathcal{B}_{k, i}: \mathbb{R}_{+}^{N(2 K-1)} \rightarrow \mathbb{R}_{+}^{N}$ $\forall k \in \mathcal{K}$, meaning that $\mathcal{B}_{k, i}\left(\mathbf{p}_{\backslash k, i} ; \mathbf{p}_{\backslash i}\right)$ is now a function of both the opponents' power allocation $\mathbf{p}_{\backslash k, i}$ in time slot $i$, and the total power allocation $\mathbf{p}_{\backslash i}$ in the other time slot $\backslash i$, with $\backslash i=2$ if $i=1$, and $\backslash i=1$ if $i=2$. Note that this notation is adopted in the remainder of the paper for mathematical convenience only, as it is straightforward to verify that $\mathbf{p}_{\backslash i}$ has no practical impact on $\mathcal{B}_{k, i}$ when $k \in\left\{\mathcal{M}_{1} \cup \mathcal{M}_{2} \cup \mathcal{S}\right\}$. An analogous modification can be applied to the mappings $t_{k, i}$, such that $t_{k, i}: \mathbb{R}_{+}^{N(2 K-1)} \rightarrow \mathbb{R}_{+} \forall k \in \mathcal{K}$.
} 
corresponds to the power allocation yielding the minimum total power expenditure when the minimum-rate constraint (17) is met with equality, under the assumption that $\mu_{k, 2} \leq \xi_{k, 2}$, with $\mu_{k, i}$ defined as in Sect. III-A, that ensures that the investigated problem is feasible.

Analogously to Sect. III-B, we can define a mapping $t_{k, 2}$ : $\mathbb{R}_{+}^{N(2 K-1)} \rightarrow \mathbb{R}_{+}$using (12), provided that $\nu_{k, 2}$ is replaced by the water level $\lambda_{k, 2}$ defined in (19).

\section{Generalized Nash equilibrium problem}

Given the best-response functions reported in the subsections above, we can now investigate the solution of the associated game $\mathcal{G}$. Observe that introducing the minimum rate constraint (17) at the relay side imposes a coupling among the feasible per-slot strategy sets $\mathcal{D}_{k, i} \subseteq \mathbb{R}_{+}^{N}$, thus making the distributed technique developed in [10] not directly usable.

The problem of finding the equilibrium of games with coupled constraints, called a generalized Nash equilibrium problem (GNEP), consists in computing the generalized Nash equilibrium (GNE) points $\mathbf{p}^{\star}$, defined as the fixed point(s) of the joint best-response function $\mathcal{B}(\mathbf{p})$, defined as

$$
\mathcal{B}(\mathbf{p})=\prod_{i=1}^{2} \prod_{k \in \mathcal{K}} \mathcal{B}_{k, i}\left(\mathbf{p}_{\backslash k, i} ; \mathbf{p}_{\backslash i}\right) .
$$

Otherwise stated, $\mathbf{p}^{\star}=\left[\left(\mathbf{p}_{1}^{\star}\right)^{T},\left(\mathbf{p}_{2}^{\star}\right)^{T}\right]^{T}$ is a GNE of $\mathcal{G}$ if and only if $\mathbf{p}^{\star} \in \mathcal{B}\left(\mathbf{p}^{\star}\right)$.

Interestingly, the equilibrium point of the game can be obtained as the solution of a QVI problem, as stated in the following proposition. For the sake of notation, let us first introduce the following definitions. For all $k \in \mathcal{K}$, let $\mathcal{P}_{k, i}$ be the set defined as

$$
\mathcal{P}_{k, i}=\left\{\mathbf{p}_{k, i} \in \mathbb{R}_{+}^{N}: \quad h_{k}\left(\mathbf{p}_{k, i}\right) \triangleq \mathbf{1}^{T} \mathbf{p}_{k, i}-P_{k, i} \leq 0\right\} .
$$

For all $k \in\{\mathcal{S} \cup \mathcal{R}\}$, let us also define the point-to-set maps

$$
\mathcal{Q}_{k, i}\left(\mathbf{p}_{\backslash i} ; \mathbf{p}_{\backslash k, i}\right)=\left\{\mathbf{p}_{k, i} \in \mathbb{R}_{+}^{N}: g_{k, i}\left(\mathbf{p}_{k, i} ; \mathbf{p}_{\backslash k, i}, \mathbf{p}_{\backslash i}\right) \leq 0\right\}
$$

where

$$
g_{k, i}\left(\mathbf{p}_{k, i} ; \mathbf{p}_{\backslash k, i}, \mathbf{p}_{\backslash i}\right) \triangleq \Psi_{k}+\mathbf{1}^{T} \mathbf{p}_{k, i}-\frac{1}{t_{k, i}^{\star}\left(\mathbf{p}_{\backslash i} ; \mathbf{p}_{\backslash k, i}\right)}
$$

with $t_{k, i}$ defined as in Sects. III-B and III-C.

Finally, let $\mathbf{p}=\left[\mathbf{p}_{1}^{T}, \mathbf{p}_{2}^{T}\right]^{T}$ be the generic strategy profile of $\mathcal{G}$, and define the set-valued function $\mathcal{D}(\mathbf{p})=\prod_{k \in \mathcal{K}} \mathcal{D}_{k}(\mathbf{p})$, where $\mathcal{D}_{k}=\mathcal{D}_{k, 1} \times \mathcal{D}_{k, 2}$, as

$$
\begin{aligned}
& \mathcal{D}_{k, 1}= \begin{cases}\mathcal{P}_{k, 1}, & \text { if } k \in\left\{\mathcal{M}_{1} \cup \mathcal{M}_{2}\right\} \\
\mathcal{P}_{k, 1} \cap \mathcal{Q}_{k, 1}\left(\mathbf{p}_{2} ; \mathbf{p}_{\backslash k, 1}\right), & \text { if } k \in \mathcal{S} \\
\mathbf{0}_{N}, & \text { if } k \in \mathcal{R}\end{cases} \\
& \mathcal{D}_{k, 2}= \begin{cases}\mathcal{P}_{k, 2}, & \text { if } k \in \mathcal{M}_{1} \\
\mathcal{P}_{k, 2} \cap \mathcal{Q}_{k, 2}\left(\mathbf{p}_{1} ; \mathbf{p}_{\backslash k, 2}\right), & \text { if } k \in\{\mathcal{S} \cup \mathcal{R}\} \\
\mathbf{0}_{N}, & \text { if } k \in \mathcal{M}_{2} .\end{cases}
\end{aligned}
$$

Proposition 1: The GNEP can be modeled as

$$
\left(\mathbf{p}-\mathbf{p}^{\star}\right)^{T} \mathbf{F}\left(\mathbf{p}^{\star}\right) \geq 0 \quad \forall \mathbf{p} \in \mathcal{D}\left(\mathbf{p}^{\star}\right)
$$

where $\mathbf{F}: \mathbb{R}_{+}^{2 N K} \rightarrow \mathbb{R}_{+}^{2 N K}$ is the vector $\mathbf{F}(\mathbf{p})$ collecting the mappings

$$
\mathbf{F}_{k, i}\left(\mathbf{p}_{k, i}, \mathbf{p}_{\backslash k, i}\right)=-\nabla_{\mathbf{p}_{k, i}} R_{k, i}\left(\mathbf{p}_{k, i}, \mathbf{p}_{\backslash k, i}\right) .
$$

Proof: Consider the QVI in (27), where $\mathbf{F}$ is a continuous mapping and $\mathcal{D}$ is a set-valued function with closed and convex images. Under these conditions, and since $\mathcal{D}$ can be described by the parametric inequalities in (22) and (24), the QVI can be reformulated as a system of constrained equations via its Karush-Kuhn-Tucker (KKT) conditions, as discussed in [13]. Thus, if a point $\mathbf{p}^{\star}$ jointly satisfies the KKT conditions of (27), then $\mathbf{p}^{\star}$ is a solution of the QVI. For the sake of briefness, we leave to the reader the easy task of verifying that the solution of the KKT system, associated to the $k$ th user operating in the time slot $i$, corresponds to the best response $\mathcal{B}_{k, i}$ whose expressions have been discussed in the previous subsections. Consequently, the power allocation vector $\mathbf{p}^{\star}$ solving (27) is a GNE of the game.

\section{Sequential PEnalty apPROACH}

A common approach to deal with coupling constraints in game theory is the definition of a pricing scheme aiming at imposing those constraints to the users in a distributed fashion [17]. In this section, we make use of the proposed QVI formulation to derive a distributed algorithm to achieve the GNE of the game.

In spite of the huge potential of the QVI modeling, relatively few studies have been devoted to the numerical solution of finite-dimensional QVIs. Among them, [18] proposes a sequential penalty approach that enables the solution of the QVI via a sequence of modified VIs, in which the mapping $\mathbf{F}$ is decreased by a penalty term.

Formally, consider the iteration $j$, and let $\left\{\rho^{(j)}\right\}$ be a sequence of positive scalars satisfying $\rho^{(j)}<\rho^{(j+1)}$ and tending to $\infty$. Define also a sequence of $2 K$-dimensional vectors $\left\{\gamma^{(j)}\right\}$ whose elements are computed as

$\gamma_{k, i}^{(j)}= \begin{cases}{\left[\gamma_{k, i}^{(j-1)}+\rho^{(j-1)} g_{k, i}\left(\mathbf{p}_{k, i}^{(j-1)} ; \mathbf{p}_{\backslash k, i}^{(j-1)}, \mathbf{p}_{\backslash i}^{(j-1)}\right)\right]^{+}} & \text {if } k \in\{\mathcal{S} \cup \mathcal{R}\} \\ 0, & \text { if } k \in\left\{\mathcal{M}_{1} \cup \mathcal{M}_{2}\right\} .\end{cases}$

For any $k \in \mathcal{K}$, the vector $\mathbf{p}^{(j)}=\left[\left(\mathbf{p}_{1}^{(j)}\right)^{T},\left(\mathbf{p}_{2}^{(j)}\right)^{T}\right]^{T} \in \mathcal{P}$, where $\mathcal{P}=\prod_{i=1}^{2} \prod_{k \in \mathcal{K}} \mathcal{P}_{k, i}$, can be then obtained as the solution of the following VIs:

$$
\left(\mathbf{p}-\mathbf{p}^{(j)}\right)^{T}\left(\mathbf{F}\left(\mathbf{p}^{(j)}\right)+\boldsymbol{\gamma}^{(j)}\right) \geq 0 \quad \forall \mathbf{p} \in \mathcal{P} .
$$

In the sequel, we will discuss the convergence property of the proposed sequential penalty approach. In this sense, a key ingredient is the continuity of the coupling constraints with respect to the set of power allocation strategies $\mathbf{p}$, as stated in the following proposition.

Proposition 2: Assuming that the maximum available powers $P_{k, 2}$, for all RSs $k \in \mathcal{R}$, guarantee the feasibility of the associated best response problems, then $g_{k, i}\left(\mathbf{p}_{k, i}, \mathbf{p}_{\backslash i} ; \mathbf{p}_{\backslash k, i}\right)$, 
with $k \in \mathcal{S} \cup \mathcal{R}$, is a continuous function with respect to all variables, and the solution of (30) converges to the GNE as $j \rightarrow \infty$.

Proof: The proposition will be proved by contradiction. First, from (24), note that $g_{k, i}$ is continuous if and only if $t_{k, i}\left(\mathbf{p}_{\backslash i} ; \mathbf{p}_{\backslash k, i}\right)$ is a continuous mapping. Then, assume that there exists a pair $(k, i)$ for which we can find two paths, $\mathbf{d}^{\prime}, \mathbf{d}^{\prime \prime} \in \mathbb{R}^{N(2 K-1)}$ such that $\mathbf{d}^{\prime} \neq \mathbf{d}^{\prime \prime}$, and

$$
\lim _{\epsilon \rightarrow 0} t_{k, i}\left(\check{\mathbf{p}}_{\backslash k, i}-\epsilon \mathbf{d}^{\prime}\right) \neq \lim _{\epsilon \rightarrow 0} t_{k, i}\left(\check{\mathbf{p}}_{\backslash k, i}-\epsilon \mathbf{d}^{\prime \prime}\right)
$$

where $\check{\mathbf{p}}_{\backslash k, i} \triangleq\left[\mathbf{p}_{\backslash i}^{T} ; \mathbf{p}_{\backslash k, i}^{T}\right]^{T}$. This implies that there exist two distinct values of the Lagrangian multiplier $\nu_{k, i}$, i.e. $\nu^{\left(\mathbf{d}^{\prime}\right)}$ and $\nu^{\left(\mathbf{d}^{\prime \prime}\right)}$, fulfilling condition (14). At the same time, we also know that $\nu_{k, i}$ represents the maximum achievable value of energy-efficient utility (7) for a given interference level. Since a fractional program with a concave numerator and a linear denominator has a unique solution, this contradicts (31), thus concluding the first part of the proof. Eventually, it can easily be verified that, under the aforementioned continuity condition, the problem at hand fulfills the hypothesis of [18, Theorem 3], which guarantees the convergence of the penalty approach as $j \rightarrow \infty$.

\section{DistRIBUTED IMPLEMENTATION}

Before studying the distributed implementation of the sequential penalty approach, let us consider the penalized VI problem (30), where $\gamma^{(j)}$ is assumed to be a fixed exogenous parameter. This problem has been largely investigated in the literature and its solution can be obtained through the simultaneous iterative waterfilling with pricing (IWFP) algorithm outlined in Table I, whose convergence is guaranteed at the condition that mapping $\mathbf{F}$ is strongly monotone [17].

In the remainder of this section, we propose a two-layer distributed algorithm in which the outer layer updates the pricing vector, while the inner layer solves the corresponding penalized VI problem via the IWFP algorithm. The design guideline for the outer-layer algorithm is provided by the following proposition.

Proposition 3: Assume that the solution $\mathbf{p}^{\star}$ of the penalized VI problem

$$
\left(\mathbf{p}-\mathbf{p}^{\star}\right)^{T}\left(\mathbf{F}\left(\mathbf{p}^{\star}\right)+\boldsymbol{\gamma}^{\star}\right) \geq 0 \quad \forall \mathbf{p} \in \mathcal{P}
$$

provides the GNE of the game $\mathcal{G}$. Then the pricing vector $\gamma^{\star}$ must solve the nonlinear complementarity problem $\mathrm{NCP}(\Phi)$, formulated as

$$
\begin{aligned}
\text { find } & \boldsymbol{\gamma} \succeq \mathbf{0} \\
\text { subject to } & \Phi(\boldsymbol{\gamma}) \succeq \mathbf{0} \\
& \gamma_{k, i}[\Phi(\boldsymbol{\gamma})]_{k, i}=0 \quad \forall k \text { and } \forall i \in\{1,2\}
\end{aligned}
$$

where the mapping $\mathbb{R}^{2 K} \rightarrow \mathbb{R}^{2 K}$ is defined as

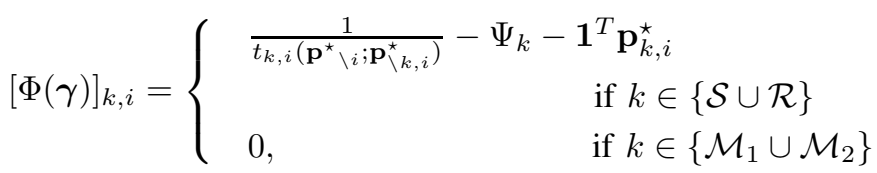

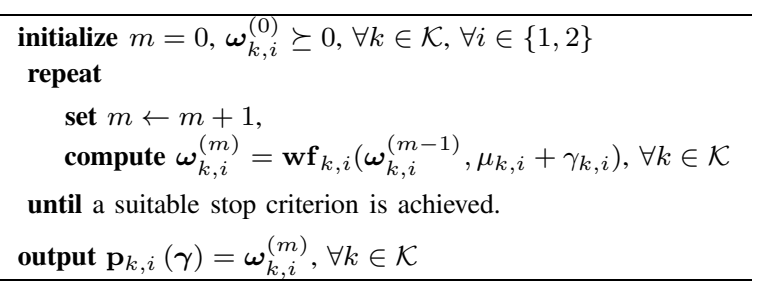

TABLE I: Simultaneous IWFP algorithm

where, for the sake of readability, the dependence of $\mathbf{p}^{\star}$ from $\gamma$ has been omitted.

Proof: Consider (32) and write down its KKT conditions $\forall k \in \mathcal{K}$ and $\forall i$ :

$$
\begin{aligned}
& \mathbf{F}_{k, i}\left(\mathbf{p}_{k}^{\star}, \mathbf{p}_{\backslash k, i}^{\star}\right)+\gamma_{k, i}^{\star}+\mu_{k, i}=\mathbf{0} \\
& 0 \leq \mu_{k, i} \perp h_{k}\left(\mathbf{p}_{k, i}\right)=0 .
\end{aligned}
$$

According to (29), we have that $\gamma_{k, i}^{\star}$ must be equal to

$$
\gamma_{k, i}^{(j)} \triangleq\left[\gamma_{k, i}^{(j-1)}+\rho^{(j)} g_{k, i}\left(\mathbf{p}_{k, i}^{(j-1)} ; \mathbf{p}_{\backslash k, i}^{(j-1)}, \mathbf{p}_{\backslash i}^{(j-1)}\right)\right]^{+}
$$

for $j \rightarrow \infty$. Now, since Proposition 2 assures the convergence of the sequential penalty approach to a bounded solution, $\gamma_{k, i}^{(\infty)}$ must be bounded too. At the same time, recalling that $\rho^{(j)} \rightarrow$ $\infty$ when $j \rightarrow \infty$ and $\gamma_{k, i}^{(j)}$ is a nonnegative scalar, we have

$$
\left[\gamma_{k, i}^{(\infty)}+\rho^{(j)} g_{k, i}\left(\mathbf{p}_{k, i}^{(\infty)} ; \mathbf{p}_{\backslash k, i}^{(\infty)}, \mathbf{p}_{\backslash i}^{(\infty)}\right)\right]^{+}<\infty
$$

only if $g_{k, i}\left(\mathbf{p}_{k, i}^{(\infty)} ; \mathbf{p}_{\backslash k, i}^{(\infty)}, \mathbf{p}_{\backslash i}^{(\infty)}\right) \leq 0$. Then, the stationarity condition (37) holds with $\gamma_{k, i}^{\star} \neq 0$ only when the coupling constraint $g_{k, i}(\cdot)$ is met with equality. Finally, by simply looking at (24), the proof follows.

Taking advantage of the result stated by Proposition 3, the following distributed algorithm is then proposed.

1) Inizialize $j=0, \mathbf{p}^{(0)} \succeq 0$, and $\boldsymbol{\gamma}^{(0)}=\mathbf{0}_{2 K}$

2) Compute $t_{k, i}\left(\mathbf{p}_{\backslash i}^{(j)} ; \mathbf{p}_{\backslash k, i}^{(j)}\right)$ using (12), $\forall k$ and $\forall i$.

3) Set $j=j+1$.

4) Compute $\mathbf{p}^{(j)}$ by solving the VI problem (30) via the IWFP algorithm depicted in Table I.

5) Compute the vector whose elements are

$$
[\tilde{\Phi}]_{k, i}=\frac{1}{t_{k}\left(\mathbf{p}_{\backslash i}^{(j-1)} ; \mathbf{p}_{\backslash k, i}^{(j-1)}\right)}-\Psi_{k}-\mathbf{1}^{T} \mathbf{p}_{k, i}^{(j)} .
$$

6) Set $\gamma_{k, i}^{(j)}=\left[\gamma_{k, i}^{(j-1)}-\rho^{(j)}[\tilde{\Phi}]_{k, i}\right]^{+}$.

7) Repeat from 2) and 6) until the maximum number of iterations is reached or a suitable stop criterion is achieved.

Finally, it is worth noting that the proposed distributed approach is equivalent to the projection method with variable steps described in [19, Algorithm 12.1.4], which provides a convergent procedure for the QVI problem whenever the hypothesis of [19, Theorem 12.1.8] are fulfilled. 


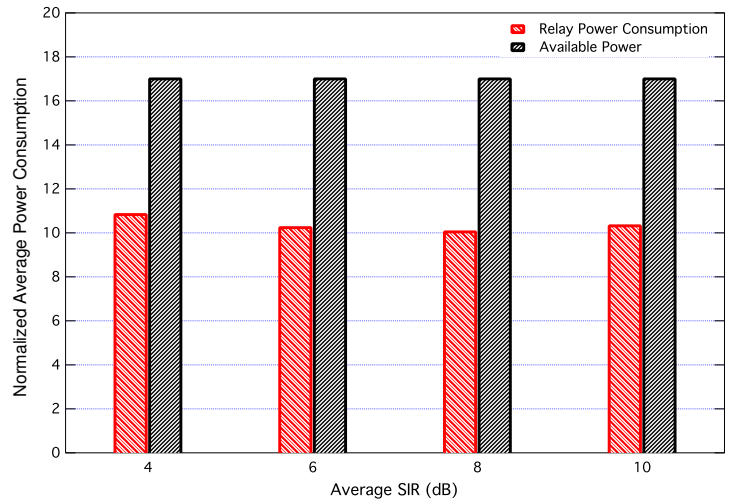

Fig. 2: Average power consumption at the relay nodes.

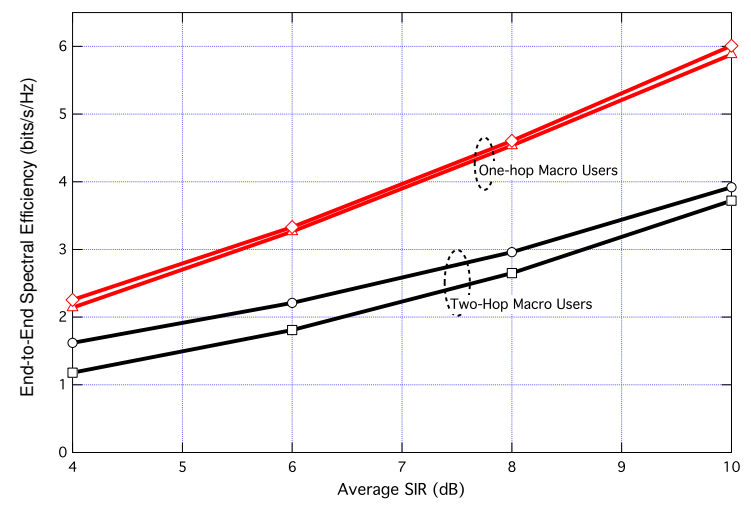

Fig. 3: MU spectral efficiency as a function of SIR.

\section{NUMERICAL RESULTS AND CONCLUDING REMARKS}

In this section, further insight into the performance of the proposed distributed algorithm is inferred via numerical simulations. The case of $S=4$ SCUs coexisting in the same geographic area with $M_{1}=2$ one-hop and $M_{2}=2$ two-hop MUs is discussed. The following system setup is used: i) the average SNR on the generic $k$ th channel defined as $\mathrm{SNR}_{k}=\mathbb{E}\left\{\left|H_{k, k}(n)\right|^{2}\right\} / \sigma_{k}^{2}(n)$ is set to $0 \mathrm{~dB}$ for the MUs and $6 \mathrm{~dB}$ for the SCUs and the RSs; ii) the average signal to interference ratio is defined as $\mathrm{SIR}_{k}=$ $\mathbb{E}\left\{\left|H_{k, k}(n)\right|^{2}\right\} /\left(\sum_{j \neq k} \mathbb{E}\left\{\left|H_{j, k}(n)\right|^{2}\right\}\right)$; iii) the interference channel is composed of $N=16$ subchannels; $i v)$ the maximum normalized power is fixed to $P_{k, i}=N$ for any active node $k$ at time step $i ; v)$ the circuit power is set to $\Psi_{k}=1$ for all $k ; v i$ ) the starting point of the distributed algorithm is the uniform power allocation strategy, i.e. $\mathbf{p}_{k, i}^{(0)}=\mathbf{1}_{N}$.

Fig. 2 presents the average power consumption of the relay nodes. Note that the relay strategy consists in maximizing their EE while guaranteeing at least the same information rate of the source-to-relay link. As apparent, at the equilibrium, this enables a consistent power saving even if a minimum rate is guaranteed to MUs. Finally, a comparison of the end-to-end spectral efficiency obtained by the MUs as function of the interference level has been reported in Fig. 3.

Conclusions: In this paper, we have developed a distributed power control strategy for relay-aided heterogeneous networks. Thanks to a proper reformulation of the optimization problem as a QVI, the transmission power of each terminal is chosen so as to maximize the preferred utility function, which can be either the information rate or the energy efficiency (possibly subject to a minimum rate constraint). By making use of a sequential penalty approach, we have demonstrated that the GNE problem associated to the proposed heterogeneous game can be solved in a distributed manner.

\section{REFERENCES}

[1] D. Feng, C. Jiang, G. Lim, L. J. Cimini, G. Feng, and G. Li, "A survey of energy-efficient wireless communications," IEEE Commun. Surveys \& Tutorials, vol. 15, no. 1, pp. 167-178, Jan. 2013.

[2] S. Verdú, "On channel capacity per unit cost," IEEE Trans. Inf. Theory, vol. 36, pp. 1019-1030, Sep. 1990.

[3] E.-V. Belmega and S. Lasaulce, "Energy-efficient precoding for multipleantenna terminals," IEEE Trans. Signal Process., vol. 59, no. 1, pp. 329-340, Jan. 2011.

[4] G. Miao, N. Himayat, and G. Y. Li, "Energy-efficient link adaptation in frequency-selective channels," IEEE Trans. Commun., vol. 58, pp. 545-554, Feb. 2010.

[5] C. Isheden, Z. Chong, E. Jorswieck, and G. Fettweis, "Framework for link-level energy efficiency optimization with informed transmitter," IEEE Trans. Wireless Commun., vol. 11, no. 8, pp. 2946-2957, Aug. 2012.

[6] J. Hoydis, M. Kobayashi, and M. Debbah, "Green small-cell networks," IEEE Veh. Technol. Mag., vol. 6, no. 1, pp. 37-43, Mar. 2011.

[7] S. Landström, A. Furuskär, K. Johansson, L. Falconetti, and F. Kronestedt, "Heterogeneous networks - increasing cellular capacity," Ericsson Review, vol. 89, 2011.

[8] K. Akkarajitsakul, E. Hossain, D. Niyato, and D. I. Kim, "Game theoretic approaches for multiple access in wireless networks: A survey," IEEE Commun. Surveys \& Tutorials, vol. 13, no. 3, pp. 372-395, 2011.

[9] G. Scutari, D. Palomar, and S. Barbarossa, "Optimal linear precoding strategies for wideband noncooperative systems based on game theory part I: Nash equilibria," IEEE Trans. Signal Process., vol. 56, no. 3, pp. 1230-1249, Mar. 2008.

[10] G. Miao, N. Himayat, G. Li, and S. Talwar, "Distributed interferenceaware energy-efficient power optimization," IEEE Trans. Wireless Commun., vol. 10, no. 4, pp. 1323-1333, Apr. 2011.

[11] I. Stupia and L. Vandendorpe, "A generalized Nash game for energy efficiency optimization: A quasi variational inequality approach," in Proc. European Signal Process. Conf., Marrakech, Morocco, Sep. 2013.

[12] A. Bensoussan, "Points de Nash dans le cas de fonctionnelles quadratiques et jeux différentiels linéaires a $N$ personnes," SIAM Journal on Control, Mar.-Apr. 1974.

[13] F. Facchinei, C. Kanzow, and S. Sagratella, "Solving quasi-variational inequalities via their KKT conditions," Mathematical Programming, pp. $1-44,2013$.

[14] R. Corless, G. Gonnet, D. Hare, D. Jeffrey, and D. Knuth, "On the Lambert W function," Advances in Computational Mathematics, vol. 5, pp. 329-359, 1996.

[15] G. Bacci, L. Sanguinetti, M. Luise, and H. V. Poor, "A gametheoretic approach for energy-efficient contention-based synchronization in OFDMA systems," IEEE Trans. Signal Process., vol. 61, no. 5, pp. 1258-1271, Mar. 2013.

[16] G. Bacci, E. Belmega, P. Mertikopoulos, and L. Sanguinetti, "Energyaware competitive link adaptation in small-cell networks," in Intl. Workshop Resource Allocation in Wireless Networks, Hammamet, Tunisia, May 2014, submitted.

[17] J.-S. Pang, G. Scutari, D. Palomar, and F. Facchinei, "Design of cognitive radio systems under temperature-interference constraints: A variational inequality approach," IEEE Trans. Signal Process., vol. 58, no. 6, pp. 3251-3271, Jun. 2010.

[18] J.-S. Pang and M. Fukushima, "Quasi-variational inequalities, generalized Nash equilibria, and multi-leader-follower games," Computational Management Science, vol. 2, no. 1, pp. 21-56, 2005.

[19] J.-S. Pang and F. Facchinei, Finite-Dimensional Variational Inequalities and Complementarity Problems, ser. Springer Series in Operations Research and Financial Engineering. New York: Springer, 2003. 